\title{
Transforming data into decision making: A spotlight review of construction digital twin
}

\author{
Yuhan LIU1, Ke CHEN², Ling MA², Shu TANG ${ }^{4}$, Tan TAN ${ }^{5}$
}

${ }^{1}$ MSc Student, Department of Construction Management, Huazhong University of Technology, Wuhan, China, 430074, Email: u201715230@ hust.edu.cn

${ }^{2}$ Associate Professor, Department of Construction Management, Huazhong University of Technology, Wuhan, China, 430074, Email: chenkecm@ hust.edu.cn, corresponding author

${ }^{3}$ Lecturer, The Bartlett School of Construction and Project Management, University College London, UK, Email: 1.ma@ucl.ac.uk

${ }^{4}$ Assistant Professor, Department of Civil Engineering, Design School, Xi'an Jiaotong-Liverpool University, Suzhou, China, 215123, Email: Shu.Tang@xjtlu.edu.cn

${ }^{5} \mathrm{PhD}$ Candidate, The Bartlett School of Construction and Project Management, University College London, UK, Email: tan.tan.17@ucl.ac.uk

\section{ABSTRACT}

The importance of digital twin (DT) has been recognized by many industries throughout the world. In the context of construction, DT and relevant technologies have been implemented at different project stages. This study first clarifies the connotation of construction digital twin (CDT) and argues the subtle but important differences between CDT and BIM. Then, this study attempts to identify the recent research and development of CDT by reviewing 46 scholarly papers published in the past three years. The review reveals that: (1) nearly $70 \%$ of the reviewed studies focus on the operation and maintenance stage, and the most popular applications of CDT include energy management, facility operation and maintenance, and structural health monitoring; (2) not all studies present a complete CDT; (3) the mechanisms to fuse different types of data for obtaining more credible decision-support information remain to be explored.

\section{INTRODUCTION}

In the era of digitalization, the concept of "digital twin (DT)" is gaining increasing popularity among many industries that are information-intensive and require real-time predictive intelligence (Chen, 2017). The construction lag behind other industries, like aerospace, manufacturing, and transportation, towards the adoption of DT, but recent years witnessed that research on DT in construction has gradually enriched.

Boje et al. (2020) reviewed the multifaceted BIM applications at the 
construction stage and highlighted the potentials of DT to address the limitations of BIM. Sacks et al. (2020) proposed a new mode for managing production in construction - digital twin construction (DTC) - that can leverage the data streaming from monitoring and artificial intelligence technologies in order to provide accurate status information and to proactively analyze and optimize the ongoing process.

With the increasing applications of DT in construction, a systematic review of recent research progress can help to evaluate existing knowledge and provide valuable references for future research. Therefore, this study will first clarify the connotation of construction digital twin (CDT) by comparing it with relevant concepts such as BIM. Then, this study will review relevant studies published in the past three years to summarize the research achievements of CDT.

\section{THE CONNOTATION OF CDT}

DT was coined 20 years ago, and its concept was getting clearer through years of research. Grieves and Vickers (2017) defined DT as a set of virtual information constructs that can fully describe a physical product from the micro atomic level to the macro geometrical level. Several studies also refer to DT as "cyber-physical integration" and believe that DT should represent the ultimate goal since no model abstraction can mirror actual things with identical fidelity (Tao et al., 2019).

By putting DT into the context of construction, a CDT basically consists of three main components, namely, a physical component that represents the physical product (i.e., building or infrastructure) and the associated construction process, a virtual component that shows the construction product and process digitally, and a connection component that connects the physical and the virtual components through data/information exchange.

A popular term related to CDT is building information modeling (BIM), which was originated from the Building Description System (BDS) in 1975. BIM is more than a $3 \mathrm{D}$ presentation of a construction project but is an indispensable information platform for making a web of decisions across the project lifecycle (Eastman et al., 2011; Chen et al., 2015).

Comparing CDT with BIM, one can find that their connotations are different. BIM focuses on a standardized semantic representation of individual building objects and the entire project, but CDT conveys a more holistic process-oriented characterization of the project that needs a connection loop between the physical project and the digital model. BIM can become, at least, a part of CDT if the model can always reflect the actual situation of the construction project. In contrast, without bi-directional data/information exchange, BIM will become "blind and deaf" to the ongoing project process and thus be far away from CDT.

\section{RESEARCH METHOD}

This study conducted a systematic review of the state-of-art of DT in construction. The database for literature search is Scopus which covers over 600 journals under the fields of "architecture", "construction and building", and "civil 
and structural engineering". The literature search was conducted on $2^{\text {nd }}$ February 2021 by using the keywords "digital twin" AND "building OR construction OR civil OR infrastructure". The language of the paper was limited to English, and the time span was set to 2018-2020. In addition, only peer-reviewed journal and conference papers were selected for further analysis.

In the second step, the authors screened the title and keywords of the collected papers and filtered suitable ones based on two criteria: (1) papers in areas irrelevant to construction, e.g., manufacturing, were excluded; (2) papers just mentioning DT but not focusing on it were excluded. After the filtering process, 46 papers were finally obtained for further analysis.

\section{RESULTS}

\section{General description of the collected literature}

As shown in Figure 1, the number of papers related to CDT has increased significantly during the three-year period from 2018 to 2020 . The number of CDTrelated papers published in 2020 has increased by 383\% compared to 2018. This result indicates that the CDT has received more and more attention from academia.

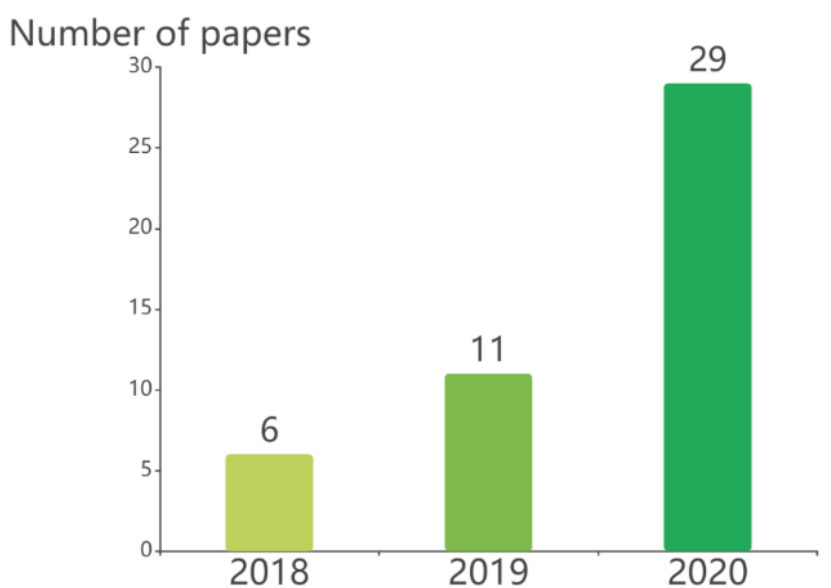

Figure 1. The number of CDT papers published between 2018 and 2020.

\section{Distribution by content category}

The 46 papers were first classified into four categories based on their main content, namely, a) conceptual framework; b) key technology; c) actual application; and d) potential illustration. After reading each paper carefully, the authors found that several papers belonged to more than one single category. For example, Austin et al. (2020) proposed a DT framework that integrated semantic modeling and machine learning. Because this paper introduced technical details of semantic modeling and machine learning, the paper should be classified into type b, i.e., technology. However, this paper also introduced a detailed case study of actual applications, which used data mining and semantic modeling to analyze the Chicago Energy Benchmarking data sets. Therefore, this paper should also be classified into type c, 
i.e., application.

As shown in Figure 2, nearly $70 \%$ of the searched papers introduced different applications of CDT. These papers will be the subject of the subsequent analysis in order to answer the following questions:

1) What project stage receives more attention;

2) What the main applications of CDT are;

3) Whether the presented CDT is a comprehensive CDT or not;

4) How CDT can help decision making.

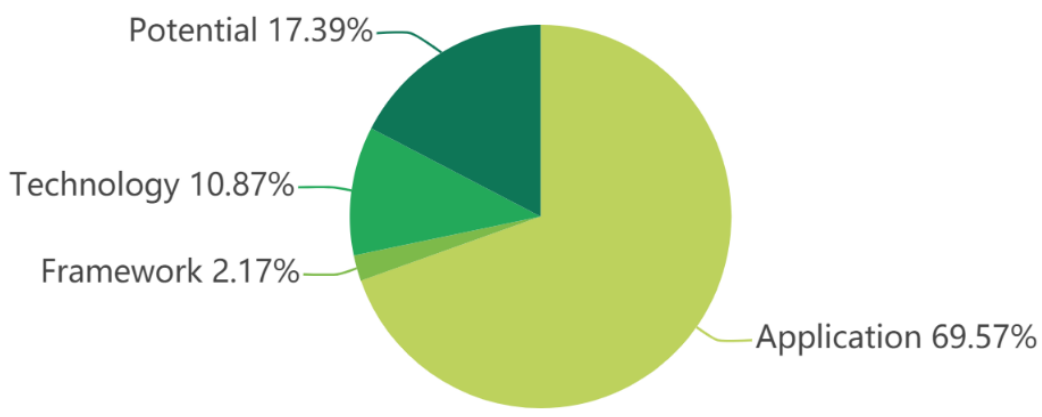

Figure 2. Content category of $\mathbf{4 6}$ papers

\section{Distribution by application stage}

Among the 32 application-type papers, $68.75 \%$ concentrated on the operation and maintenance stage, and much fewer papers focused on other stages (see Figure 3). Possible reasons could be that it is more feasible and practical to develop the DT of a building or infrastructure once the project is complete without any further changes. In comparison, at the design stage, there is generally less physical object that requires digital representation; at the construction stage, the situation is complex and dynamically change, and thus requires much more effort to update the digital model constantly.

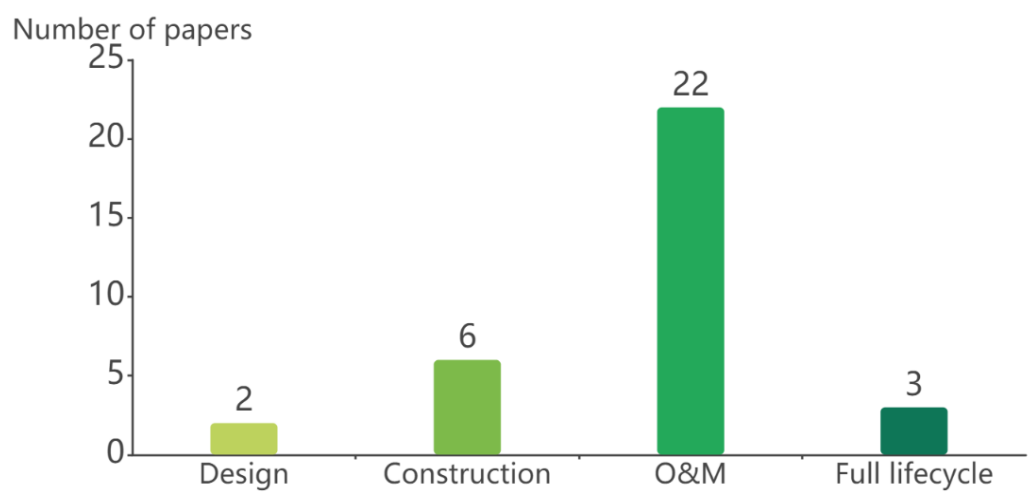

Figure 3. Distribution of the 32 application-type papers (By application stage)

\section{Distribution by application}

As can be seen from Figure 4, the top three CDT applications include energy management, facility operation and maintenance, and structural health monitoring. 
Peng et al. (2020) introduced the operation of a hospital building project, which used DT to manage the energy consumption, allocate the space, and support response to emergency. For papers introducing CDT applications at the design and construction stages, Lydon et al. (2019) presented simulation methods for the thermal design of a heating and cooling system that was integrated with a lightweight roof structure. Greif et al. (2020) proposed the use of DT to improve silo dispatch and replenishment activities for supply chain optimization.

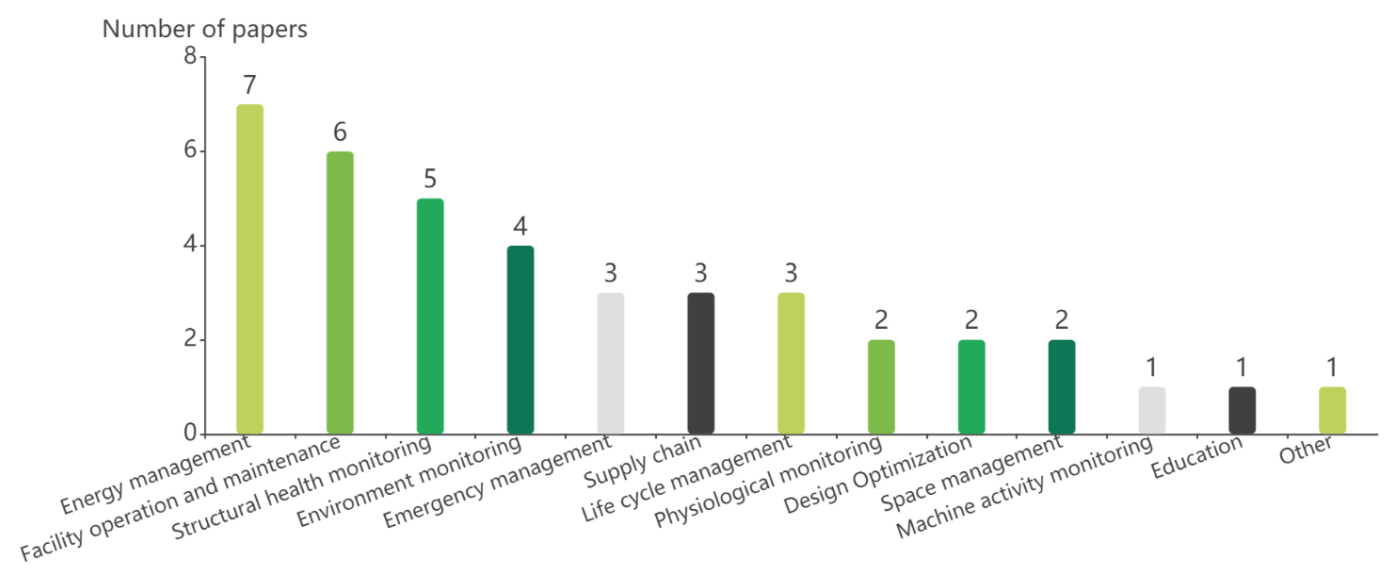

Figure 4. Distribution of the 32 application-type papers (By application)

\section{Integrity of CDT}

Based on the connotation of CDT in Section 2, the authors identified that not all the 32 papers presented a comprehensive CDT (see Figure 5). In seven papers, some important parts of CDT were omitted, and a few of them considered CDT and BIM to be interchangeable terms. One example is Kaewunruen et al. (2018) that proposed a DT-based evaluation of net-zero energy buildings for existing buildings. Although this paper established a building model through BIM and used the model for energy analysis, the subsequent lack of data input from the physical part to the virtual part. Another example is Chen and Whyte (2020), in which a digital-design structure matrix from DT to identify interdependencies and change design was presented, but the need for real-time data input from the physical to the virtual was not discussed.

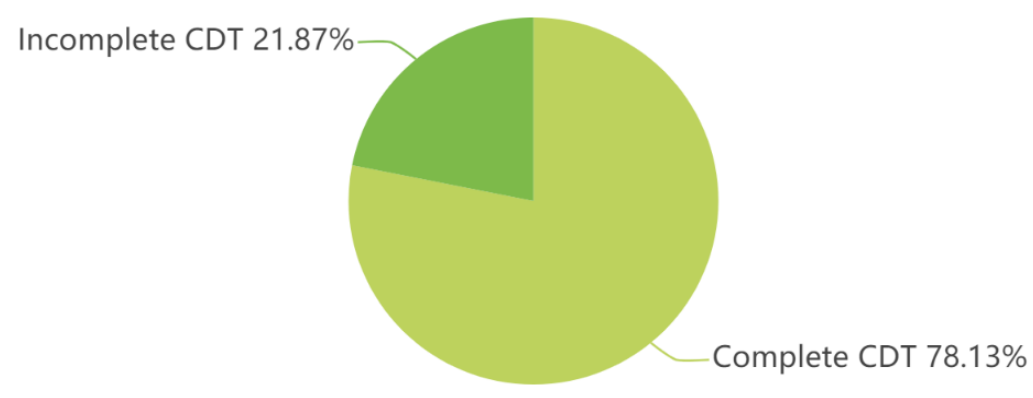

Figure 5. Integrity of CDT 


\section{Benefits of CDT}

The reviewed papers demonstrated that CDT can support different levels of information visualization, processing, and quantitation for various decision-making tasks.

CDT can visualize all necessary information of the physical construction to the end-users. For example, Chen and Whyte (2020) presented a case of the Thames Tideway Tunnel in London, showing a DT-driven design structure matrix to analyze the interdependencies of complex engineering systems. They completed asset level digital design structure matrix through BIM and used the BIM Views and the Query engine in BIMserver to read and visualize the IFC file of the project.

In terms of information processing, Kim and Ham (2020) proposed using drones and DT models to analyze utility poles. Their method estimated the maximum inclination angle of the utility pole through the image of the utility pole collected by the drone. Then, the analysis results were input into the digital model that can help determine the priority of maintenance of the utility pole. This example only used image and geographic information of utility poles, and the rules for data-driven decision support were relatively simple. More complex applications can be found in the case of the West Cambridge Campus (Lu et al., 2020a; Lu et al., 2020b; Xie et al., 2020). For instance, anomaly detection of pumps, not only did the sensor measure pump vibration data, but the building management system integrated pump on duty flag bit and other types of data into external asset operation information to identify contextual anomalies. In addition, there were temperature sensors that detected indoor temperature. If data about the indoor temperature was abnormal, the pump failure event would be queried in the facility management system to determine whether the detected temperature abnormality is caused by asset failure related issues.

When more data become available in CDT, data mining can be performed to discover new knowledge from large data sets. This benefit has been illustrated in a few reviewed papers. For instance, Fan et al. (2020) proposed a disaster city digital twin, which has three sources of data: remote sensing, social sensing, and crowdsourced collection. Their proposed DT platform used artificial intelligence to integrate and analyze the multi-sourced data and extract valuable information for situation assessment, disaster management optimization, disaster relief operations, and other emergency management practices. However, the integration and usage of multiple types of data in CDT are still at the immature stage and need further research.

\section{CONCLUSION}

This study analyzes recent research achievements in CDT by conducting a systematic review of the literature published between 2018 and 2020. The review found that the research on CDT showed an upward trend. Most of the reviewed studies focused on structural health monitoring, facility operation and maintenance, and energy management at the operation and maintenance stage. However, the review also found that, as there is no universal definition of CDT, some researchers 
have deviations in their cognition of this concept and suggest that BIM and CDT could be interchangeable.

It should be noted that, depending on the application, CDT can deal with different types of data input and output. The most straightforward benefit of CDT is to visualize real-time data and information to end-users. Additionally, CDT can integrate different types of data from both the physical and the virtual to obtain more value-added information.

The continued development of the CDT will bring fresh vitality to the construction industry. Based on the review findings, the authors suggested that future research should explore more applications of CDT, especially for the construction stage. In addition, future research can propose strategies to help address technical and managerial challenges in the deployment of CDT in actual projects.

\section{ACKNOWLEDGMENTS}

This study is supported by the Major Scientific and Technological Innovation Project in Hubei Province (2020ACA006).

\section{REFERENCES}

Austin, M., Delgoshaei, P., Coelho, M., and Heidarinejad, M. (2020). “Architecting smart city digital twins: combined semantic model and machine learning approach." Journal of Management in Engineering, 36(4), 04020026.

Boje, C., Guerriero, A., Kubicki, S., and Rezgui, Y. (2020). "Towards a semantic Construction Digital Twin: Directions for future research." Automation in Construction, 114, 103179.

Chen, K., Lu, W., Peng, Y., Rowlinson, S., and Huang, G. Q. (2015). "Bridging BIM and building: From a literature review to an integrated conceptual framework." International Journal of Project Management, 33(6), 1405-1416.

Chen, L., and Whyte, J. (2020). "Analysing interdependencies of complex engineering systems using a digital twin-driven design structure matrix." Construction Research Congress 2020: Computer Applications (pp. 417-426). Reston, VA: American Society of Civil Engineers.

Chen, Y. (2017). "Integrated and intelligent manufacturing: perspectives and enablers." Engineering, 3(5), 588-595.

Eastman, C., Teicholz, P., Sacks, R., and Liston, K. (2011). BIM handbook: A guide to building information modeling for owners, managers, designers, engineers and contractors. John Wiley \& Sons.

Fan, C., Zhang, C., Yahja, A., and Mostafavi, A. (2020). "Disaster City Digital Twin: A vision for integrating artificial and human intelligence for disaster management." International Journal of Information Management, 56, 102049. 
Greif, T., Stein, N., and Flath, C. M. (2020). "Peeking into the void: Digital twins for construction site logistics." Computers in Industry, 121, 103264.

Grieves, M., and Vickers, J. (2017). "Digital twin: Mitigating unpredictable, undesirable emergent behavior in complex systems." Transdisciplinary perspectives on complex systems (pp. 85-113). Springer, Cham.

Kaewunruen, S., Rungskunroch, P., and Welsh, J. (2018). "A digital-twin evaluation of net zero energy building for existing buildings". Sustainability, 11(1), 159.

Kim, J., and Ham, Y. (2020). "Vision-Based Analysis of Utility Poles Using Drones and Digital Twin Modeling in the Context of Power Distribution Infrastructure Systems." Construction Research Congress 2020: Computer Applications (pp. 954-963). Reston, VA: American Society of Civil Engineers.

Lu, Q., Parlikad, A. K., Woodall, P., Don Ranasinghe, G., Xie, X., Liang, Z., Konstantinou, E., Heaton, J., and Schooling, J. (2020). "Developing a digital twin at building and city levels: case study of West Cambridge campus." Journal of Management in Engineering, 36(3), 05020004.

Lu, Q., Xie, X., Parlikad, A. K., and Schooling, J. M. (2020). "Digital twin-enabled anomaly detection for built asset monitoring in operation and maintenance." Automation in Construction, 118, 103277.

Lydon, G. P., Caranovic, S., Hischier, I., and Schlueter, A. (2019). "Coupled simulation of thermally active building systems to support a digital twin." Energy and Buildings, 202, 109298.

Peng, Y., Zhang, M., Yu, F., Xu, J., and Gao, S. (2020). "Digital Twin Hospital Buildings: An Exemplary Case Study through Continuous Lifecycle Integration." Advances in Civil Engineering, 2020.

Sacks, R., Brilakis, I., Pikas, E., Xie, H. S., and Girolami, M. (2020). "Construction with digital twin information systems." Data-Centric Engineering, 1.

Tao, F., Qi, Q., Wang, L., and Nee, A. Y. C. (2019). "Digital twins and cyberphysical systems toward smart manufacturing and industry 4.0: Correlation and comparison." Engineering, 5(4), 653-661.

Xie, X., Lu, Q., Rodenas-Herraiz, D., Parlikad, A. K., and Schooling, J. M. (2020). Visualised inspection system for monitoring environmental anomalies during daily operation and maintenance." Engineering, Construction and Architectural Management, pp. 1835-1852.

The published version of this paper is available in the ASCE Library at: https://doi.org/10.1061/9780784483848.033 